\title{
Era for New Paradigm
}

\section{Won H Kim ${ }^{\star}$}

Department of Biochemistry, Yonsei University Wonju College of Medicine, Wonju, Kangwondo 220-701, Korea

\section{Paradigm Shift}

Science pursues truth. However, scientific quest for truth is confined in the frame of a paradigm. Gödel mathematically proved that for any system, there will always be statements that are true, but that are unprovable within the system. There always appear facts which cannot be explained with current paradigm. Such fact can be judged only at higher dimension. Paradigm shift is unavoidable.

Paradigm shift is initiated from unexplainable fact with current paradigm. Quantum science came from the trial to explain the unexplainable fact, black body radiation. $21^{\text {st }}$ century needs new paradigm. New paradigm will be initiated from unexplainable fact. This time it could be water memory.

\section{Homeopathy}

The story of water memory begins from homeopathy. Homeopathy uses toxic substance to enhance the ability of natural healing. It is thus very natural that homeopathy tried dilution method with physical stimulation to reduce harmful effect of toxin. What homeopathy found is that even though the poisonous substance gets diluted to the point where the molecule is no longer exist, its effect still remained, which could mean 'water memory'. Even though it has been used successfully clinically for over 200 years, homeopathy has been ignored by the orthodox medical circles until now because current science could not explain how a substance's effect can be shown without actual substance.

\section{Scientific Approach Begins from Benveniste}

Although French scientist Benveniste did not know about homeopathy, he was the first to show water's memorizing ability in scientific way. In 1988 Benveniste and colleagues published a controversial article showing a biological reaction of ultra-high diluted solution ( $10^{-120}$ of its original density) [1], which could be called as 'water memory'. As biological reaction in the absence of any effective molecules cannot be explained by conventional theory focusing only on the water structure of which the hydrogen bonds last only picoseconds scale.

The results of Belon et al. sparked investigations with various seriousness. The most serious one was the research performed double blind by 4 independent European laboratories in 2004 [2]. The interesting fact was the purpose of their double blind test was to disprove Benvensite's research but instead proved that it was in fact true.

\section{Recent Approaches}

Water memory could be reproduced in many other laboratories. P53 is a DNA binding protein and functions as a potent tumor suppressor. However, there is virtually no practical way to utilize the function of P53 clinically. If the molecular structural information of P53 (expressed as information wave from now on) could be transferred to water or any medium contacting water, various strategies could be possible. We have devised information wave transferring device using $7.8 \mathrm{~Hz}$, resonance frequency of earth. The device could activate and transfer the information wave of the matter to and any other medium. The water to which information wave of P53 was transferred actually inhibited cancer proliferation, showed anti-metastasis and increased apoptosis. The results have been published at the first issue of Journal of Vortex Science and Technology [3].

Nobel laureate Montagnier and colleagues showed that DNA polymerase could even recognize information wave of specific DNA sequence transferred to water and produce new DNA copies [4], suggesting that information wave of DNA is the physical entity. They also used similar device using $7.8 \mathrm{~Hz}$ frequency to transfer the information wave of DNA to water. The existence of DNA information wave as physical entity in space was also shown in so called 'phantom DNA effect' by Poponin [5]. He showed that diffraction pattern of DNA which appear by laser radiation could be regenerated even after DNA sample was removed.

\section{From Pilot Wave to Information Wave}

Benveniste thought that when a wave propagated from molecule is transferred to a cell receptor through water, the wave can induce resonance of a receptor initiating intracellular signal transmission. The inherent wave of matter is not a new concept. In 1924 de Broglie proposed that every matter has accompanying wave. De Broglie further suggested that the wave inherent to matter is guiding the trajectory of particle, and called it as pilot wave. In 1961 Eisberg showed by calculation that pilot wave is much faster than the speed of light. Calling such wave as 'information wave' as it decrease thermodynamic entropy (this editorial is using same terminology), Tiller suggested mass particle and pilot wave (information wave) interacts so as to be experimentally operational. According to Tiller, information wave of the matter could be transferred to water by physical stimulation as in homeopathy, and the information wave transferred to water could affect receptor by resonance, and intracellular signal transmission could be initiated [6].

\section{Digital Biology}

Benveniste further showed that water memory could be recorded to computer by passing white noise through the solution. The recorded and digitized sound file could be transferred through email to induce physiological reaction in the far distance [7]. As biological reaction could be regenerated even from the computer recorded digitized signal, he developed his theory under the name Digital Biology. Concepts of Digital Biology inducing physiological reaction without the presence of any material and digital recording/transmission of the substance' wave could be reproduced in other laboratories.

*Corresponding author: Won H. Kim, Department of Biochemistry, Yonse University Wonju College of Medicine, Ilsandong 162, Wonju, Kangwondo 220-701, Korea, Tel: 82-33-741-0283; Fax: +82-33-743-0411, E-mail: kimhwbio@yonsei.ac.kr

Received October 23, 2013; Accepted October 24, 2013; Published October 26 2013

Citation: Kim WH (2013) Era for New Paradigm. Fluid Mech Open Acc 1: e105. doi: 10.4172/2090-8369.1000e105

Copyright: (C) $2013 \mathrm{Kim} \mathrm{WH}$. This is an open-access article distributed under the terms of the Creative Commons Attribution License, which permits unrestricted use, distribution, and reproduction in any medium, provided the original author and source are credited. 


\section{New Approaches Using Digitized Information Wave}

Benveniste used white noise to pick up the information wave of the substance. Recently we have used information wave transferring device and strong light source to digitize information wave of substance. Information wave recorded in computer could be expressed into a visual shape in two dimensional space. Digitized information wave in $2 \mathrm{D}$ space showed the same characteristic as the original substance, which is much easier to use and has wider applications.

This 2D form of information wave can be also used for transfer of information of the original substance in a way of printing to plastics or other flat materials. How could a form in 2D space elicit the same biological reactions as original matter? If information wave is faster than the speed of light as shown by calculation, it should have negative mass. According to Dirac, space is described as a domain of negative energy and negative mass. It may be possible that digitized information wave expressed on plastic card is connected with the information wave of the original substance through holographic space itself to form the information wave of the original substance around the card.

Digitized information wave expressed in $2 \mathrm{D}$ space could be used various ways. A card containing digitized information wave of medically effective substance such as drug or hormone could be carried close to human body to induce physiological effect. Digitized information wave of the substance could be modulated to electricity. This suggests that every electrical device could be used as a generator of specific information wave of medically effective substance. Information wave of the substance modulated to water, plastic card, and electricity could help us maintain health just by changing the environment. The results with digitized information wave expressed in $2 \mathrm{D}$ space will be published in Journal of Vortex Science and Technology.

\section{Vortex Science}

We started from water memory and end up with digitized information wave in 2D space which could be modulated to even electricity. It is like that food as information wave of the substance could be contained in a variety of dishes; water, space and electricity. Substance itself might be one of the containers for carrying the information wave of the substance (maybe the best container).

So far in this article I have assumed the existence of inherent wave part of the substance (expressed as information wave) which could be separated by physical stimulation and exist independently in water and functions like the substance. In contrast, instead of inherent wave portion of matter, Meyl suggested that every wave including electromagnetic wave could be rolled to vortex and act like particle. According to Meyl, everything is in wave state with only differences in the shape of the wave whether it is open or closed form (vortex), in which particle state is a highly condensed vortex, and thus, particle and wave state of single substance does not coexist, although their proportion is in equilibrium as a whole. Vortex theory of Meyl could also explain water memory, of which closed form of wave from the matter (vortex state) shows particle-like characteristic inside water [8].

\section{Journal of Vortex Science and Technology}

As was shown in this article, there are countless facts which cannot be explained with conventional paradigm. What is needed is the scientific breakthrough like Plank did 100 years ago to explain black body radiation. Current paradigm views world as materialism. Every biological reaction occurs by physical contact between molecules. If we scale the size of the cell as big city like Seoul where more than 10 million people live, the size of each molecule almost corresponds to each person. The meeting between two people without prior appointment when and where would be almost impossible. The biological reaction by physical contact between molecules cannot explain the fast reaction of living body. Now we have seen that biological reactions could occur without presence of materials. There are enough facts which require new paradigm beyond materialism. Many scientists who cannot be satisfied with current paradigm are pursuing the new science. That's what we expect from Journal of Vortex Science and Technology.

\section{References}

1. Davenas E, Beauvais F, Amara J, Oberbaum M, Robinzon B, et al. (1988) Human basophil degranulation triggered by very dilute antiserum against IgE. Nature 333: 816-818.

2. Belon P, Cumps J, Ennis M, Mannaioni PF, Roberfroid M, et al. ( 2004) Histamine dilutions modulate basophil activation. Inflamm Res 53: 181-188.

3. Won H Kim (2013) New Approach Controlling Cancer Water Memory. Jour Vort Sci and Tech 1: 1-4

4. Montagnier L, Aissa J, Del Giudice E, Lavallee C, Tedeschi A (2011) DNA waves and water. J. Phys.: Conf. Ser. 306012007.

5. Poponin V (2011) The DNA PHANTOM EFFECT: Direct Measurement of A New Field in the Vacuum Substructure. TWM.

6. http://www.tiller.org/

7. Benveniste J, Jurgens P, Hsueh W, Aissa J (1997) Transatlantic transfer of digitized antigen signal by telephone link. Journal of Allergy and Clinical Immunology 99: S175

8. Meyl (2001) Scalar waves Theory and Experiments. Journal of Scientific Exploration 15: 199-205 